\title{
IMMUNOMODULATORS AS THERAPEUTIC AGENT
}

\author{
*Neelottama Kushwaha, Swatantra K.S. Kushwaha \\ Department of Pharmacy, Pranveer Singh Institute of Technology, Kanpur, INDIA-208020 \\ *Corresponding Author's Email: neelottama@yahoo.co.in
}

Received 12 May 2012; Review Completed 23 May 2012; Accepted 13 July 2012, Available online 15 July 2012

\begin{abstract}
ABS TRACT:
Immunomodulators are substances that have been shown to modify the immune systems response to a threat upon it. They modulate and potentiate the weapons of your immune system keeping them in a highly prepared state for any threat it may encounter. With this balancing effect, all subsequent immune responses improve. When your immune system is in this highly prepared state, the invading organisms do not have the time to build up force and strength before the immune system attacks destroys and/or weakens the invader.
\end{abstract}

Key words: Immunomodulators, psoriasis, Crohn's disease, Systemic Mycoses

\section{INTRODUCTION:}

The modulation of immune system activity is a required pharmacological intervention for many medical conditions. Immunosuppression is integral to the treatment of autoimmune diseases and allergies, and the inhibition and prevention of immune responses also constitutes a prominent treatment aim for transplant recipients. Conversely, immune system stimulation is adopted for a number of infections and cancers. With traditional immunomodulatory drugs continuing to exhibit limited efficacy and adverse side effects, the drive to develop new, improved, and specifically targeted immunotherapy.

The modulation of immune system activity is a required pharmacological intervention for many medical conditions. Immunosuppression is integral to the treatment of autoimmune diseases and allergies, and the inhibition and prevention of immune responses also constitutes a prominent treatment aim for transplant recipients. Conversely, immune system stimulation is adopted for a number of infections and cancers. With traditional immunomodulatory drugs continuing to exhibit limited efficacy and adverse side effects, the drive to develop new, improved, and specifically targeted immunotherapies has been gaining momentum. ${ }^{1}$

\section{DIS EAS ES PATTERN IN WHICH IMMUNO- MODULATORS USE:}

\section{Immunomodulators in the treatment of psoriasis:}

Psoriasis is a common skin disorder characterized by erythematous scaly plaques. Recently, it has been realized that psoriasis is an inflammatory T-cell mediated disorder. Many recent therapeutic advances are attempting to control the expression of psoriasis by suppressing T-cell mediated inflammation.

Cyclosporine and related drugs, which are effective in the treatment of psoriasis, was the key to the development of the concept that psoriasis is an immune mediated disorder. These therapies demonstrably reduce the number of activated T-lymphocytes, which correlates with clinical remission. Monoclonal antibodies directed against key components of the inflammatory process have been studied in an attempt to produce safer, more effective and selective immunosuppressive agents. ${ }^{2}$

Langerhans cell maturation (activation)

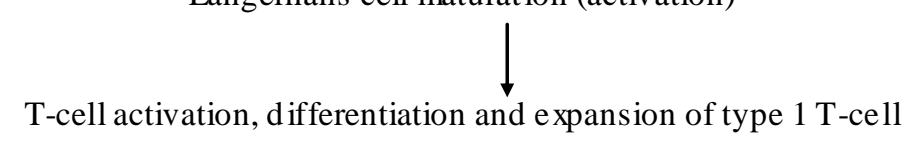

T-cell activation, differentiation and expansion of type $1 \mathrm{~T}$-cell

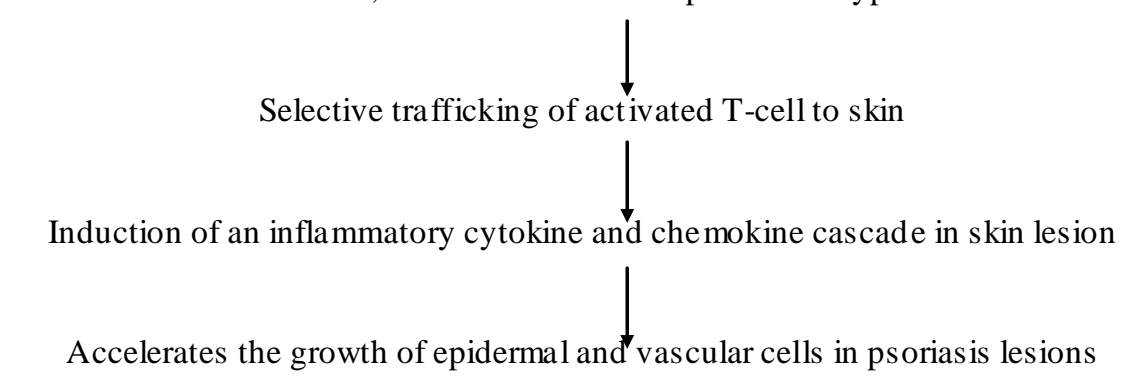

Figure 1: Critical steps in the immunopathogenes is of psoriasis and sites of intervention of biologic therapeutics 


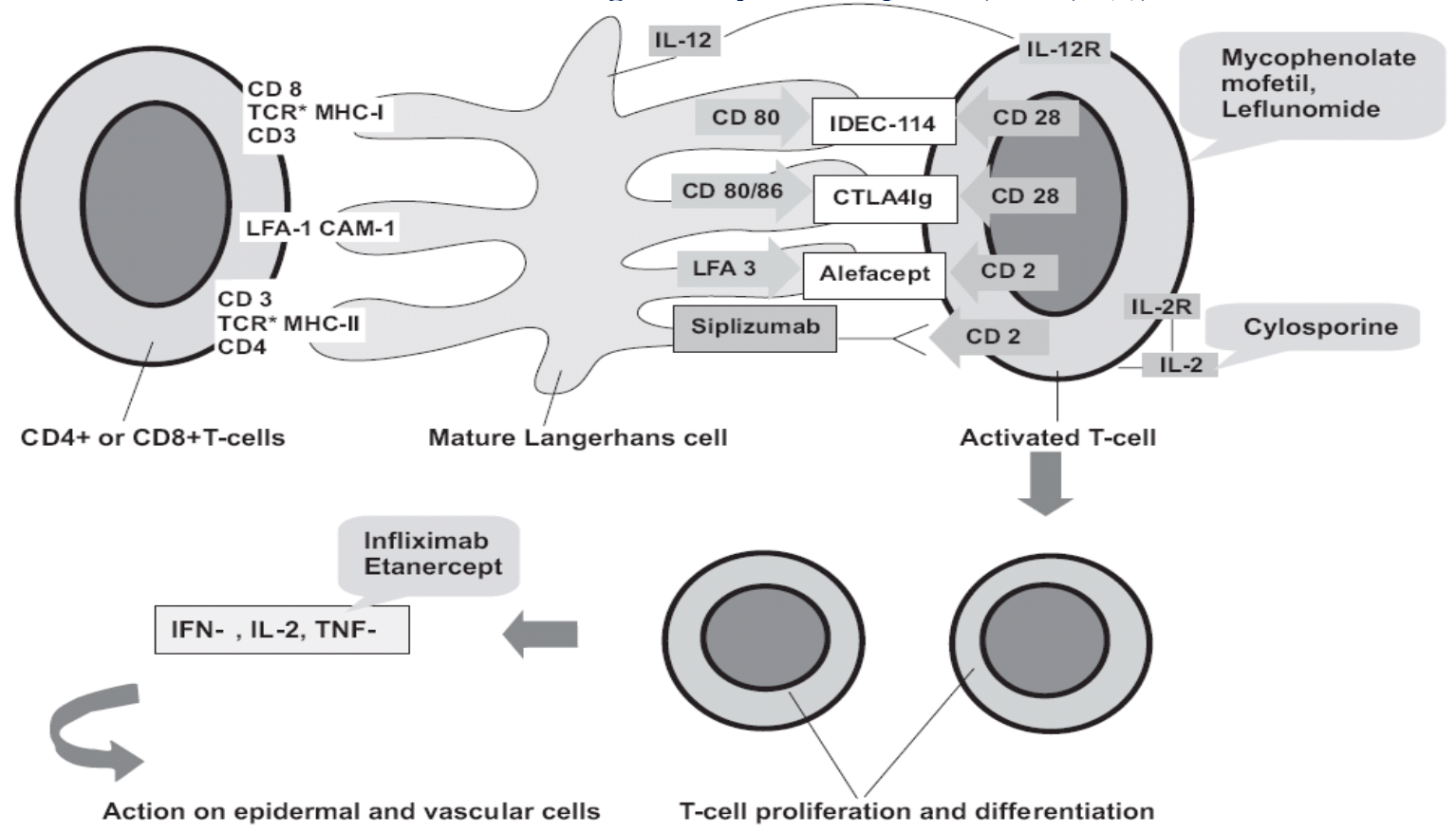

Figure 2: Molecules as sociated with T-cell activation (site of action of mycophenolate mofetil and leflunomide), proliferation and differentiation ${ }^{2}$

A T-cell interacts with a mature Langerhans cell to become activated as shown in figure 2 . Here the various receptors involved andcytokines released are the sites of action of various biologics (TCR stands for T-cell receptor, MHC-I and II for major histocompatibility complex molecules on antigen presenting cells [Langerhans cells], LFA-1 for lymphocyte function-associated antigen-1, LFA-3 for lymphocyte function-associated antigen-3, ICAM-1 for intercellular adhesion molecule-1, IL-12 for interleukin-12, IL-2 for interleukin-2 [inhibited by cyclosporine], IL-12R for interleukin-12 receptor, IL-2R for interleukin-2 receptor [inhibited by cyclosporine], CTLA4 for cytotoxic $\mathrm{T}$ lymphocyte antigen-4, IFN- $\gamma$ for interferon-gamma, TNF- $\alpha$ for tumor necrosis factor-alpha [blocked by infliximab or etanercept], CD8, CD3, CD4, CD80, CD86, LFA3, CD40, CD 28, CTLA4, CD2, CD40L [site of action of monoclonal based therapies] are various receptors on $\mathrm{CD}+$ cells and Langerhans cells.

Cyclosporine is a powerful immunosuppressive drug with no appreciable effect on the bone marrow.

Leflunomide has demonstrated antiviral, antitumorigenic, and immunosuppressive properties. Its active metabolites reversibly inhibit the mitochondrial enzyme dihydroorotate dehydrogenase, the rate-limiting step in the de novo synthesis of pyrimidines. As a result, it inhibits autoimmune T-cell proliferation and production of autoantibodies by Bcells. Its clinical uses include rheumatoid arthritis, psoriasis vulgaris and psoriatic arthropathy, bullous autoimmune disorders, Wegener's granulomatosis and systemic vasculit is.

Immunomodulators for Crohn's disease and ulcerative colitis:

The first two immunomodulators to be used widely in IBD are azathioprine (Imuran 1, Azasan1) and 6- mercaptopurine (6-MP, Purinetholi), drugs that are chemically quite similar. They are used to maintain remission in Crohn's disease and ulcerative colitis. Both have a slow onset of action (three to six months for full effect). Accordingly, they are usually given along with another faster-acting drug (such as corticosteroids).

Other immunomodulators to treat IBD are cyclosporine A and tacrolimus, both used for organ transplantation as well. Cyclosporine A has a more rapid onset of action (one to two weeks) than azathioprine and 6-MP. It is useful in people with active Crohn's disease, but only when given intravenously and at high doses. Both cyclosporine A and tacrolimus have been more effective in treating people with severe ulcerative colit is, and are generally given until one of the slower-acting immunomodulators begins to work or until the patient undergoes curative surgery. Tacrolimus can be used in Crohn's disease when corticosteroids are not effective or when fistulas develop. ${ }^{3}$

Methotrexate (MTX®, Rheumatrex ${ }^{\circledR}$, Mexate $\AA$ ) works more rapidly than azathioprine or 6-MP, and is given by weekly injections. It is an effective option for people with Crohn's disease who have not responded to other treatments and cannot tolerate other immunosuppressants.

\section{Immunomodulators in Treating Multiple Sclerosis :}

\section{Approved Therapies:}

The approval of three interferons-beta (IFN $\beta$ ) and of glatiramer acetate (GA) by the US Food and Drug Administration (FDA) in the early 1990s was the most important recent advance in multiple sclerosis (MS) therapy. These immunomodulators are now a first-line treatment of relapsing-remitting (RR) and relapsingprogressive (RP) MS. In 2000, mitoxantrone - a cytolytic immunosuppressant - was approved for the treatment of patients with a rapidly progressive disease. A second immunosuppressant - natalizumab (Tysabri®) - was approved in November 2004. Given its remarkable efficacy, natalizumab appeared a promising candidate for the long-term treatment of RR MS. However, severe adverse events led to a temporary suspension of the drug 


\section{Neelottama et al}

J ournal of Drug Deli ve

(February 2005 to June 2006). After its reintroduction to the market, natalizumab was reserved for carefully selected patients under strict surveillance and for a limited period of time. Immunomodulators are well tolerated for years, but their efficacy is relatively modest. Immunosuppressants are more effective, but their toxicity prevents long-term treatments. Numerous clinical trials investigate new molecules in order to improve the efficacy of immunomodulators and reduce the toxicity of immunosuppressants. ${ }^{4}$

\section{Experimental Therapies:}

\section{FTY720 (Fingolimod $®)$}

Fingolimod ${ }^{\circledR}$ converts endogenous sphingosine into its phosphate form, which is a high-affinity agonist of the sphingosine 1 (S 1P1) receptor. Fingolimod first accelerates homing of lymphocytes to lymph nodes and then blocks egress of lymphocytes from lymphoid tissues. The lymphocyte sequestration in lymphoid tissues leads to a major decrease in $\mathrm{T}$ - and particularly B-cell count (25\% of baseline values) in peripheral blood. Fingolimod also causes thymocyte apoptosis, inhibits vascular endothelial growth factor (VEGF)-induced brain capillary leakage, impairs dendritic cell trafficking and induces a shift from Th1 (pro-inflammatory) to Th2 (anti-inflammatory) cytokine production. It has been observed in animal experiments that Fingolimod strongly reduces transmigration of macrophages into brain parenchyma.

\section{Rituximab:}

Rituximab is a monoclonal Ab binding to the CD20 surface molecule that induces a selective, long-lasting depletion of B cells that is only partially reconstituted after one year.

\section{BBR2778 (Pixantrone $®)$ ):}

Pixantrone ${ }^{\circledR}$ is an analogue of MX without severe cardiotoxicity. In addition, its weaker DNA-constant binding and its lower stimulation of topoisomerase IImed iated DNA alterations suggest a lower risk of TRAL.

\section{Alemtuzumab (Campath-1H®):}

Alemtuzumab, a monoclonal Ab targeting the surface CD52 molecule mainly expressed on T lymphocytes and monocytes, is a potent and selective immunosuppressant of cellular immunity leading to a complete deletion of lymphocytes. Monocytes and B cells return to normal within three months, but T-cell depletion persists for up to five years. ${ }^{5}$

\section{Immunomodulators in Systemic Mycoses:}

Various biopotent molecules have been studied for their potential to modulate and restore impaired immune functions required to resist fungal infections. These include recombinant human cytokines including granulocyte colony stimulating factor (rHuG-CSF), recombinant human macrophage colony stimulating factor (rHuM-CSF), interferons etc., some of which have shown encouraging results. Among the various categories of immunomodulating agents reported so far, certain peptides seem to hold better promise36. Six novel peptides viz., hexapeptide (89/215), glycopeptides (89/729, 90/341), pentapeptide (SP-5) and lipopeptides (86/450, 84/201),
\& Therapeutics; 2012, 2(4), 51-60

synthesized have been evaluated in CDRI for potent immunostimu lant activity. ${ }^{6}$

\section{Immunotherapy of Cancer:}

The active immunotherapeutic approach to treatment of cancer is specific and based upon the premise that tumor antigen are immunogenic and the host is sufficiently immunocompetent to mount an effective immune response to an autologous tumor. Theoretically, a weak or suppressed host immune system that had allowed the formation of a tumor may be overridden by active immunization and or immunostimulation. In practice, vaccines composed of so-called autologous tumor extract have been used to treat patient with malignant melanoma and purified melanoma tumor-associated antigens have been used to elicit antibody response in melanoma patients.

The specificity of immune mechanisms could be harnessed against tumor cells. This might use the patient's own immune system (immunostimulants) the transfer of antibodies (i.e. passive immunization) or $\mathrm{T}$ cells from an outside source. Ideally, these agents would be targeted to molecules expressed on the cancer cells but not on healthy cells. However, such tumor-specific antigens have been hard to find, and so many of the immune agents now in use do target healthy cells as well, but the hope is that these can later be replaced. ${ }^{7}$

\section{Immunostimulants:}

There is considerable evidence that cancer patients have $\mathrm{T}$ cells that are capable of attacking their tumor cells. In fact, it may be that the appearance of cancer is a failure of immune surveillance: the ability of one's own immune system to destroy cancer cells as soon as they appear.

Immunostimulants are nonspecific agents that tune-up the body's immune defenses. There have been some successes with:

- Injecting adjuvant-like agents directly into the tumor. The only one that succeeds often enough to remain in use is the bacterial preparation BCG. Introduced into the bladder, it can help eradicate early-stage bladder tumors.

- Oral therapy with levamisole, a drug widely-used for deworming (people as well as animals), has been used to treat a variety of cancers but with inconsistent results.

- $\quad$ interleukin-2 (IL-2), a potent growth factor for T cells;

- alpha-interferon (IFN- $\alpha$ )

\section{Cancer Therapy with Monoclonal Antibodies}

A number of monoclonal antibodies show promise against cancer, es pecially cancers of white blood cells (leukemias, ly mphomas, and multiple myeloma).

\section{Some examples:}

Rituximab (trade name $=$ Ritu xan $\left.{ }^{\circledR}\right)$. Used to treat B-cell lymphomas. The CD20 molecule to which it binds is present on most B-cells, healthy as well as malignant, but over the months following treatment, new healthy B cells are formed from precursors that do not have CD20 and thus were not destroyed by the treatment. ${ }^{8}$ 
Neelottama et al

J ournal of Drug Deli very \& Therapeutics; 2012, 2(4), 51-60

Herceptin $®$. Binds HER2, a growth factor receptor found on some tumor cells (some breast cancers, lymphomas). The only monoclonal so far that seems to be effective against solid tumors.

Alemtuzumab (MabCampath $\left.{ }^{\circledR}\right)$. Binds to CD52, a molecule found on white blood cells. Has produced complete remission of chronic lymphocytic leukemia (for 18 months and counting).

Lym-1 (Oncolym®). Binds to the HLA-DR-encoded histocompatibility antigen that can be expressed at high levels on ly mphoma cells.

Bevacizumab (Avastin $\left.{ }^{\circledR}\right)$. Binds to vascular endothelial growth factor (VEGF) thus blocking its action and depriving the tumor of its blood supply.

Cetu ximab (Erbitu $\left.{ }^{\circledR}\right)$. Used to treat colorectal cancers.

Ipilimumab. Blocks the immunosuppresive effects of CTLA-4. Has shown some benefit against metastatic melanoma.

\section{Immunotoxins}

A major problem with chemotherapy is the damage the drugs cause to all tissues where rapid cell division is going on. What is needed is a "magic bullet", a method of delivering a cytotoxic drug directly and specifically to tumor cells, sparing healthy cells. Such a magic bullet would have two parts:

- a monoclonal antibody specific for the cancer cell attached to

- a cytotoxic drug or toxin that kills the cell once it gets inside.

Many such conjugates have been tried but only two have found their way into anticancer therapy.

\section{Mylotarg®:}

- A monoclonal antibody that binds CD33, a cellsurface molecule expressed by the cancerous cells in acute myelogenous leukemia (AML) but not found on the normal stem cells needed to repopulate the bone marrow.

- calicheamicin, a complex oligosaccharide that makes double-stranded breaks in DNA.

Mylotarg® is the first immunotoxin to show promise in the fight against cancer.

\section{CAT-3888 (formerly known as B L22):}

- a monoclonal V-region antibody fragment against CD22, a molecule found on the surface of B-cell leukemias and ly mphomas, with

- Pseudomonas exotoxin, a bacterial product that blocks protein synthesis in cells causing them to self-destruct by apoptosis.

\section{Radi oi mmunother apy}

Monoclonal antibodies against tumor antigens can also be coupled to radioactive atoms. The goal with these agents is to limit the destructive power of radiation to those cells (cancerous) that have been "fingered" by the attached monoclonal antibody.
Examples: Zevalin®. This is a monoclonal antibody against the CD20 molecule on B cells (and lymphomas) conjugated to either the radioactive isotope indium-111 $\left({ }^{111} \mathrm{In}\right)$ or the radioactive isotope yttriu m- $90\left({ }^{90} \mathrm{Y}\right)$. Both are given to the lymphoma patient, the ${ }^{111}$ In version first followed by the ${ }^{90} \mathrm{Y}$ version (in each cases supplemented with Rituxan). Bexxar® (tositumomab). This is a conjugate of a monoclonal antibody against CD20 and the radioactive is otope iodine-131 $\left({ }^{131} \mathrm{I}\right)$. It, too, is designed as a treatment for lymphoma. Although both Bexxar ${ }^{\circledR}$ and Zevalin ${ }^{\circledR}$ kill normal B cells, they don't harm the B-cell precursors because these do not express CD20. So, in time, the precursors can repopulate the body with healthy $B$ cells.

\section{Cancer Therapy with T Cells}

Tumor destruction is done by cells. Antibodies may help, but only by identifying the cells to be destroyed, e.g., by macrophages. But $\mathrm{T}$ cells, like cytotoxic $\mathrm{T}$ ly mphocytes (CTL), are designed to destroy target cells.

\section{Using a Bispecific Monoclonal Antibody}

Blinatumomab is a synthetic monoclonal antibody each arm of which carries a binding site with a different specificity:

- one arm binds to CD19, an antigen found on the surface of B cells and B-cell ly mphomas;

- The other arm binds to CD3, a cell-surface molecule on T cells, including cytotoxic T ly mphocytes (CTLs).

By forming a bridge between CD3 and CD19, blinatumomab is able to attach $\mathrm{T}$ cells to $\mathrm{B}$ cells and activate the $\mathrm{T}$ cells to kill the $\mathrm{B}$ cells.

Early clinical trials with blinatumo mab on a small nu mber of patients appear quite promising. Modest doses of the drug produced partial and, in a few cases, complete regression of their ly mphoma.

\section{Allografts of $T$ cells}

After total destruction of the patient's own white blood cells ("myel oablative condi tioning")

One approach to curing leukemia (and some other cancers) is to treat the patient with such high doses of chemotherapy and radiation that the leukemic cells are killed. Unfortunately, such high doses also destroy the patient's bone marrow. If the patient is to survive the treatment, he or she must be given a transplant of hematopoietic stem cells — the cells from which all blood cells are formed.

The stem cells can be:

- An autograft; that is, from bone marrow harvested from the patient and stored before treatment begins. In this case, however, the marrow must also be treated to purge it of all cancer cells it may contain before it is returned to the patient. This sometimes fails.

- An allograft; that is, cells harvested from another person, usually a family member sharing the same major histocompatibility molecules.

Allografted hematopoietic stem cells also sometimes fail to cure, but in that case it is because not all of the patient's 


\section{Neelottama et al}

Journal of Drug Delive

leukemic cells were destroyed. However, an infusion of T lymphocytes from the blood of the same donor that provided the cells can finish off the job. This effect is called the graft-versus-leukemia effect. However, most (if not all) of the donor T cells are probably attacking normal cell surface molecules, not tumor-specific ones. (Even if the donor and recipient are matched for the major histocompatibility molecules, there will be minor ones that elicit a rejection response.) So the patient may also suffer life-threatening graft-versus-host disease (GVHD).

\section{Autografts of $T$ cells: Tumor-Infiltrating Lymphocytes} (TIL)

Solid tumors contain lymphocytes that are specific for tumor antigens. For many years, Steven A. Rosenberg and his associates at the U. S. National Cancer Institute have tried to enlist these cells in cancer therapy.

On September 19, 2002, he reported his most promising results to date. The procedure:

- Isolate $\mathrm{T}$ cells - both $\mathrm{CD}^{+}{ }^{+} \mathrm{T}$-helper cells and $\mathrm{CD} 8^{+}$ cytotoxic $\mathrm{T}$ lymphocytes (CTL) from samples of the tumor (melanoma)

- Test them in vitro to find the most efficient killers of the melanoma cells.

- Grow large numbers of them in culture (using the powerful T-cell growth factor IL-2).

- Treat the patient with modest doses of cytotoxic drugs to reduce - but not destroy - the bone marrow.

- Reintroduce the mix of Th cells $\left(\mathrm{CD} 4^{+}\right)$and CTL $\left(\mathrm{CD}^{+}\right)$into the patient (along with IL-2).

The results:

- The infused cells usually took up longterm residence.

- In 10 of 13 patients, their melano ma cells — including all metastases — regressed either partially or completely.

\section{Autografts of gene tically-modified $\mathrm{T}$ cells}

On April 20, 2006, the Rosenberg group reported some success with melanoma patients using a modification of the TIL procedure.

The patient's $\mathrm{T}$ cells were removed and treated with a retroviral vector containing the $\alpha \beta$ TCR specific for a me lano ma antigen. Large numbers of these were grown in culture. After partial myeloablative conditioning to "make room" for them, the genetically-modified lymphocytes were infused into the patient. This application of gene therapy succeeded in eliminating the metastases and providing a disease-free period of two years in two patients.

\section{Cancer Vaccines}

Any response of the patient's own immune system immune surveillance - has clearly failed in cancer patients. The purpose of cancer vaccines is to elicit a more powerful active immunity in the patient. Several approaches are being explored. y \& Therapeutics; 2012, 2(4), 51-60

\section{Dendritic-Cell Vaccines}

Dendritic cells are the most potent antigen-presenting cells. They engulf antigen, process it into peptides, and "present" these to T cells.

\section{Tumor-Antigen-S pecific Vaccines}

These vaccines are used to immunize the patient with an antigen universally expressed by tumors of that type (but not by normal cells) mixed with some form of adjuvant that will enhance the response.

For example, there is growing evidence that many cancer patients mount an immune response - both antibodymediated and cell-mediated - against the tumor antigen designated NY-ESO-1. Deliberate immunization with this protein (plus an adjuvant) boosts this response and has shown some promise in early clinical trials. (Cells in the testis do not express HLA antigens, so are not at risk from attack by NY-ESO-1-specific cytotoxic T lymphocytes).

Immunomodulation in organ-transplantation; Advances in surgical techniques, availability of more selective immunosuppressant, careful patient selection, and proper post-surgery management are major factors in making transplantation the treatment of choice for end-stage organ failure.

Non-specific immunosuppressive therapy in an adult patient is usually cyclosporine, started intravenously at the time of transplantation, and given orally when feeding is tolerated. Typically, methylprednisone is started also at time of transplantation, and then reduce to a maintenance dose. Azathioprine may also be used in conjunction with prednisone to achieve adequate immunosuppression. The objective of immunosuppression is to protect the transplant, but general immunosuppression or excessive immunosuppression may lead to undesirable complication, e.g. opportunistic infections and potential malignancies. These adverse effects would be avoided if selective immunosuppression could be achieved. A number of Fungal Immunosuppressives have been isolated from fermentation broths and demonstrated to have immunotherapeutic efficacy. The known and most commonly used is cyclosporine. Recently, two other fungal metabolites, sirolimus, previously known as rapamycin and FK-506 are in various stages of development. Sirolimus marked ly suppresses IL-2 or IL-4 driven $\mathrm{T}$ cell proliferation. The preclinical studies suggest that sirolimus is a potent immuno-suppressive agent in transplantation and autoimmune disease models. FK-506 interferes with IL-2 synthesis and release, and cyclosporine like profile, but is considerably more potent in vitro with IC50 value approximately 100 fold lower. Mycophenolate mofetil, Brequinar and Deoxyspergualin are in various phases of clinical evaluation. Identification of the therapeutic efficacy and safety will be important factors in determination of utility as immunosuppressive agents.

\section{Treatment of keloids and hypertrophic scars:}

Hypertrophic scars and keloids may follow local skin trauma or inflammatory skin disorders like laceration, tattoos, burns, injections, ear-piercing, vaccination, bites, 
acne, abscess or surgery. They are the consequences of uncontrolled synthesis and deposition of dermal collagen.

The first step in minimizing scarring should be attention to the early care of wounds. Following recommendations are based on general principles of wound healing (Table 1). The goal with minor wounds such as abrasions is to achieve rapid epithelization by moist healing with ointment or semiocclusive dressings. When epithelization is delayed beyond 10-14 days the incidence of hypertrophic scarring goes up dramatically. Surgical closure of an open wound should take into account the tension on the wound. Wounds subjected to tension due to motion, body location, or losses of tis sue (after excision of a lesion) are at increased risk of scar hypertrophy and spreading. Appropriate splinting of the tissue with permanent intradermal sutures should be considered. A useful technique is a subcuticular closure with a polypropylene suture that can be left in place for six months.

Table 1: Treatment option for keloids and hyper trophic scar

Pressure garments
Radiation
Superficial X-ray
Electron beam therapy
Interstitial radiotherapy
Excision
Intralesional injection
Triamcinolone
5-flurouracil
Bleomycin
Cryotherapy
Silicon gel dressings

Biologic Therapy for Inflammatory B owel Disease:

Potential options for the medical management of inflammatory bowel disease (IBD), particularly Crohn's disease, are increasing at an unprecedented rate. In addition to infliximab, secondgeneration anti-tumor necrosis factor (TNF)-alpha agents such as adalimumab* and certolizumab* have shown efficacy in the induction and maintenance of remission in patients with Crohn's disease. Optimizing these medications with respect to timing of initiating therapy and use of concomitant immunomodulators is a topic of great debate. Other potential agents include antibodies targeting alternate mechanis ms of inflammation, such as natalizumab* and visilizumab*. The excitement over these emerging agents is tempered by the potential toxicity, some of which is unique to each class of drug. ${ }^{10}$

\section{Recently de veloped Immunomodulators:}

Imiquimod a recently developed imidazoquinolin heterocyclic amine belongs to a new class of drug, immune response modifier agents, which can be attributed to the induction of a number of cytokines including interferonalpha (IFN-alpha), interferon-gamma (IFN-gamma), tu mor necrosis factor-alpha, interleukins-1, $-6, \quad-8, \quad-12$. Imiquimod increases in interferon and tumor necrosis factor in cultures of cells isolated from animal skin, and increases interleukin- 8 concentrations in human keratinocyte and fibroblast cultures. Although in vitro studies have shown that imiquimod has no direct antiviral effects, the drug does exhibit antiviral and antitumor effects in vivo through induction of human cytokines. Although imiquimod cream recently became available for the treatment of genital and perianal warts, the topical mechanism of action of imiquimod is not yet fully understood. It is believed that stimulation of local cytokines by imiquimod leads to a reduction in human papilloma virus (HPV) load; to wart regression and to the normalization of keratinocyte proliferation without evidence of scarring. Treatment with topical $5 \%$ imiquimod cream has shown promising results in the treatment of genital warts in immunocompetent individuals. Furthermore, it is an interesting alternative therapeutic method for other cutaneous viral skin lesions among immunocompetent and HIV-in fected patients.

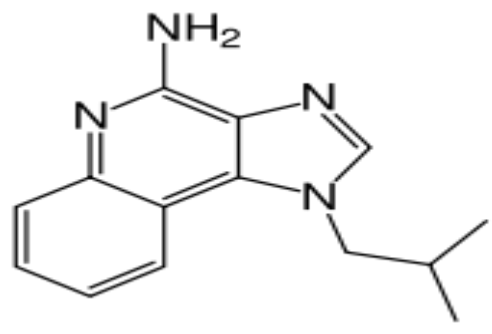

Imiquimod

The exact mechanism of action in which imiquimod and its analogs activate the immune system is not yet known. Nevertheless, it is known that imiquimod activates immune cells by ligating the toll-like receptor 7 (TLR7), commonly involved in pathogen recognition, on the cell surface. Cells activated by imiquimod via TLR-7 secrete cytokines (primarily interferon- $\alpha$ (IFN- $\alpha$ ), interleukin- 6 (IL-6) and tumor necrosis factor- $\alpha(\mathrm{TNF}-\alpha)$ ). There is evidence that imiquimod, when applied to skin, can lead to the activation of Langerhans cells, which subsequently migrates to local lymph nodes to activate the adaptive immune system. Other cell types activated by imiquimod include natural killer cells, macrophages and Bly mphocytes. 11

Thalidomide and its immunomodulatory (IMiDs) analogs (lenalidomide, Revlimid, CC-5013; CC-4047, ACTIMID) are a novel class of compounds with numerous effects on the body's immune system, some of which are thought to mediate the anticancer and anti-inflammatory results observed in humans. Thalidomide is currently being used experimentally to treat various cancers and inflammatory diseases. It is approved for the treatment of dermal reaction from leprosy and is currently in phase III trials for multiple myeloma. Thalidomide and IMiDs inhibit the cytokines tumor necrosis factor- $\alpha$ (TNF- $\alpha)$, interleukins (IL) $1 \beta, 6,12$, and granulocyte macrophagecolony stimulating factor (GM-CSF). They also costimulate primary human $\mathrm{T}$ ly mphocytes inducing their proliferation, cytokine production, and cytotoxic activity thereby increasing the $\mathrm{T}$ cells' anticancer activity. They induce an IL-2-mediated primary $\mathrm{T}$ cell proliferation with a concomitant increase in IFN- $\gamma$ production and decrease the 
Neelottama et al

Journal of Drug Deli very \& Therapeutics; 2012, 2(4), 51-60

density of TNF- $\alpha$-induced cell surface adhesion molecules ICAM-1, VCAM-1, and E-selectin on human umbilical vein endothelial cells. Thalidomide stimulates the Th-1 response increasing IFN- $\gamma$ levels while CC-4047 increased IL-2 as well. Some of the above immunomodulatory activities along with anti-angiogenic, anti-proliferative, and pro-apoptotic properties are thought to mediate the IMiDs'antitumor responses observed in relapsed and refractory multiple myeloma and some solid tumor cancers. This has led to their use in various oncology clinical trials. The second generation IMiD, lenalidomide, has shown potential in treating the bone marrow disorders myelodysplastic syndrome and multiple myeloma. It is currently in phase II and III trials for these diseases respectively with numerous phase II trials in other hematologic and solid tu mors. ${ }^{12}$

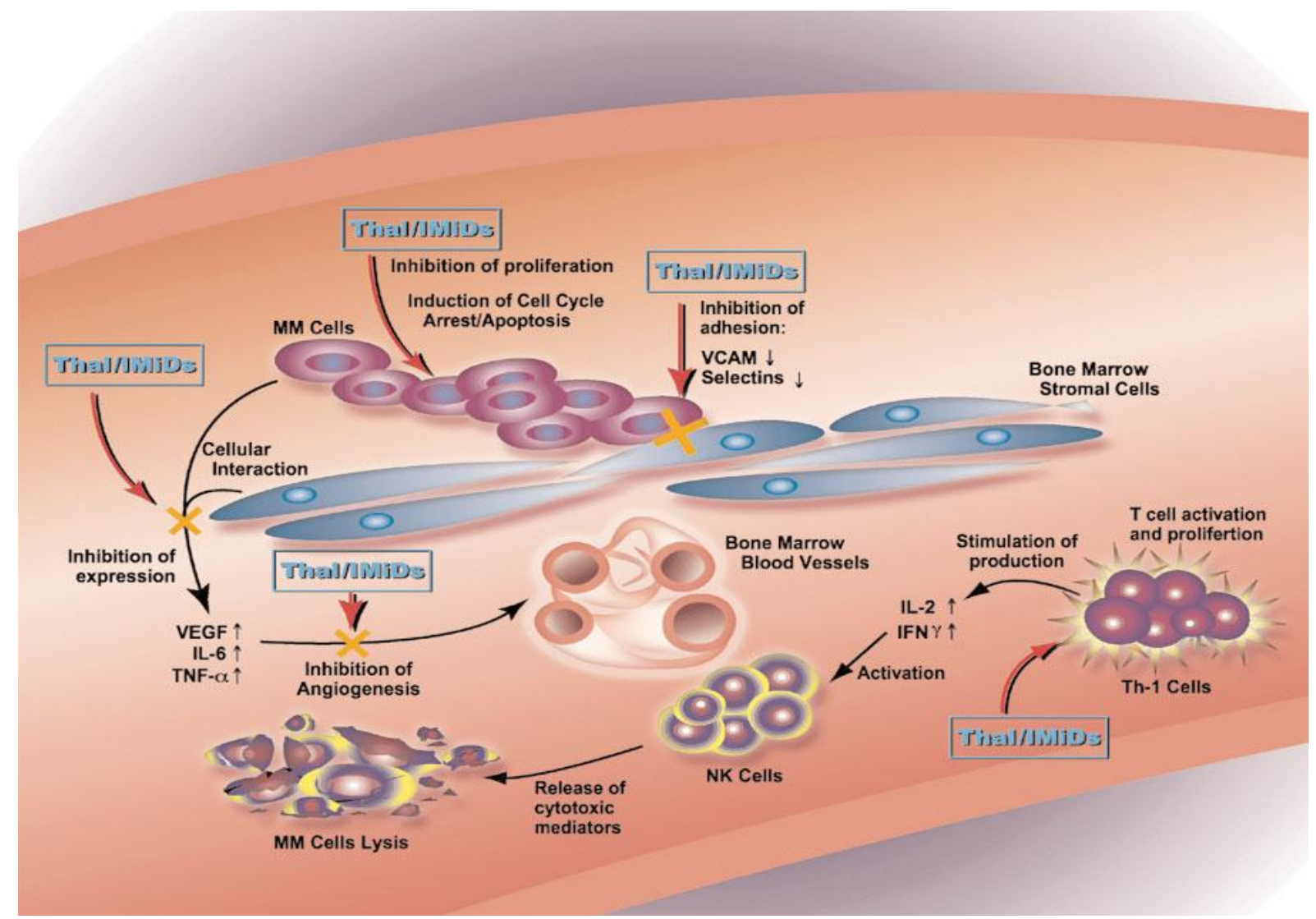

Figure 4: Sites of activity of thalido mide and IMiDÒs in the bone marrow of mu ltiple myelo ma Patients ${ }^{12}$<smiles>O=C1CCC(N2C(=O)c3ccccc3C2=O)C(=O)N1</smiles><smiles>Nc1cccc2c1CN(C1CCC(=O)NC1=O)C2=O</smiles><smiles>Nc1cccc2c1C(=O)N(C1CCC(=O)NC1=O)C2=O</smiles>

Figure 5: Structures of thalido mide, lenalido mide and CC-4047

9-fluorenon-4-carboxamides has been prepared as asymmetrically-substituted analogues of the antiviral tilorone and their antiherpetic and cytokine-inducing properties have been explored. The best pharmacological profiles were shown by carboxamides 1, 4, 9, and 10 endowed with anti-HSV-2, IFN $\alpha$, IFN $\gamma$ and TNF $\alpha$ - 
Neelottama et al

J ournal of Drug Deli very \& Therapeutics; 2012, 2(4), 51-60

distilled triethylamine, in dichloromethane for $24 \mathrm{~h}$. An improvement of hydrosolubility was due to amines endowed with different basic chains bearing $\mathrm{OH}$ groups or nitrogen and oxygen atoms which help in DNAintercalation or to stabilize it.<smiles>[R]NC(=O)c1cccc2c1-c1ccccc1C2=O</smiles>

$1-10$

Scheme

Table 3: 9 -flurenon-4-car boxami des

\begin{tabular}{cccc}
\hline Comp. & $\mathrm{R}$ & Comp. & $\mathrm{R}$ \\
\hline 1 & $-\left(\mathrm{CH}_{2}\right)_{2} \mathrm{OH}$ & $\mathbf{6}$ & $-\left(\mathrm{CH}_{2}\right)_{2} \mathrm{~N}\left(\mathrm{CH}_{3}\right)_{2}$ \\
2 & $-\left(\mathrm{CH}_{2}\right)_{3} \mathrm{OH}$ & 7 & $-\left(\mathrm{CH}_{2}\right)_{3} \mathrm{~N}_{\left(\mathrm{CH}_{3}\right)_{2}}$ \\
3 & $-\mathrm{CH}_{2} \mathrm{CHOHCH}_{3}$ & $\mathbf{8}$ & $-\left(\mathrm{CH}_{2}\right)_{2} \mathrm{~N}_{\left(\mathrm{CH}_{2} \mathrm{CH}_{3}\right)_{2}}$ \\
4 & $-\left(\mathrm{CH}_{2}\right)_{4} \mathrm{OH}$ & $\mathbf{9}$ & $-\left(\mathrm{CH}_{2}\right)_{2}$ \\
5 & $-\left(\mathrm{CH}_{2}\right)_{2} \mathrm{O}\left(\mathrm{CH}_{2}\right)_{2} \mathrm{OH}$ & $\mathbf{1 0}$ & $-\left(\mathrm{CH}_{2}\right)_{3}$
\end{tabular}

A new series of tilorone like compounds, based on various substitutions in position 4 on the 9-fluorenone skeleton, has been proposed as potential anti-HSV-2 agents. Synthes is and chemicalphysical characterization have been coupled with a conformational analysis focusing on the tricyclic surface exposed to the solvent and potentially able to make intercalation in the DNA duplex.

Moreover the pharmacological evaluation demonstrated that carboxamides 1, 4, 9 and 10, inducing cytokines such as IFN- $\gamma$, IFN- $\alpha$, and TNF- $\alpha$, play beneficial roles against virus replication. ${ }^{13}$

Murabutide, has been found to enhance the host's nonspecific resistance to bacterial and viral infections, to induce colony-stimulating activity, and to be well tolerated by humans. In contrast to most other exogenous immunomodulators, Murabutide is apyrogenic, does not induce inflammatory reactions and has the capacity to synergize with selected therapeutic cytokines to drive the release of T-helper 1 cytokines. Moreover, the coadministration of Murabutide with alpha interferon (IFN-a) or with interleukin-2 (IL-2), was found to dramatically enhance the antitumor activity of either cytokine, as well as the antiviral and anti-inflammatory effects of IFN-a. Furthermore, Murabutide presents a highly interesting immunopharmacological profile with its ability to regulate macrophage function. ${ }^{14}$

The mechanis ms of action of Murabutide: In conclusion, several independant studies and others allow us to determine the mechanism of biological activities of the immuno modulator Murabutide.

Interaction of the non-specific immunomodulator Murabutide with human leukocytes results in the regulation of cellular genes leading to:

- Enhanced resistance to viral and bacterial infections
- Inhibition of viral replication in infected cells

- Potentiation of antiviral, antitumor and antiinflammatory activities of therapeutic cytokines (IFNalpha; IL-2)

- Restoration of defective antigen-presenting cell function in macrophages and dendritic cells thereby leading to efficacious adaptive immunity

- Restoration of selective cytokine milieu which drives protective Th-1 responses against intracellular infections and tumor

Regulated cellular factors in Lymphocytes (HIV related):

- Inhibition of the expression of c- myc: the cellular factor required for the nuclear transport of HIV preintegration complexes

- Inhibition of the expression of RH116: a novel RNA helicase necessary for HIV transcription

- Induction of the release of HIV-suppressive chemokines.

Regulated cellular factors in macrophages (HIV-related):

- Selective induction/activation of transcription factors capable of inhibiting HIV-1 LTR.

- Induction of the release of HIV-suppressive beta chemokines.

- Inhibition of the expression of HIV-1 co-receptors CXCR4 and CCR5.

Regulated cellular factors mediating other biological effects of Murabutide:

- Up-regulation of HSP 70 as mediator of adjuvant effect. 
- Induction of IL-1 receptor antagonist, IL-1 receptor accessory protein, and soluble TNF receptors as med iators of the anti- inflammatory activity.

- Induction of BMP-8 (bone morphogenetic protein 8) and IGFBP-5 (Insulin- like growth factor binding protein 5) as protential mediators of accelarated bone formation.

- Induction of NINJ-1 and LICAM as mediators of nerve regeneration.

- Up-regulation of Toll- like receptors and LRP-5 as mediators of enhanced innate immunity against viral and bacterial infections .

- Activation of transcription factors stat-1 and IRF-2 as mediators of Th-1 responses and enhanced antiviral activity of IFN-alpha.

- Up-regulation of CD40, MHC-II, CD80 and CD86 as receptors implicated in dendritic cell- $\mathrm{T}$ cell interaction, leading to efficacious adaptive immune responses.

Leflunomide is a medication of the DMARD type, used in active moderate to severe rheumatoid arthrit is and psoriatic arthritis. It is a pyrimidine synthesis inhibitor.

Leflunomide is a pyrimidine synthesis inhibitor belonging to the DMARD (disease-modifying antirheumatic drug) class of drugs, which are chemically and pharmacologically very heterogeneous. The chemical name for leflunomide is $\mathrm{N}$-(4'-trifluoromethylphenyl)-5methylisoxazole-4-carboxamide. Leflunomide is an immunomodulatory drug inhibiting dihydroorotate dehydrogenase (an enzyme involved in de novo pyrimidine synthesis). Genuine antiproliferative activity has been proven. Additionally, several experimental models (both in vivo and in vitro) have demonstrated an anti-inflammatory effect. This double action is supposed to slow progression of the disease and to cause remission/relief of symptoms of rheumatoid arthritis and psoriatic arthritis such as joint tenderness and decreased joint and general mobility in human patients. ${ }^{15}$

\section{Contraindications and Precautions}<smiles>Cc1oncc1C(=O)Nc1ccc(C(F)(F)F)cc1</smiles>

Lefluno mide

Leflunomide has a great number of absolute and relative contraindications, in part associated with its mode of action:

- Hypersensitivity to the drug or to inactive ingredients (e.g., lactose).
- Important contraindications are preexisting pregnancy, or women of childbearing potential not using reliable anticonceptive methods.

- Preexisting significant liver or renal disease and moderate to severe diseases of the bone marrow or immune system preclude the use of Leflunomide.

- Moderate to severe bacterial, fungal or viral infections (e.g., AIDS, latent HIV-Infection, pneumonia, active tuberculos is).

- Combination with methotrexate, other DMARDs and alcohol.

\section{Side-Effects :}

The side-effects of Arava affect quite a number of organ systems, are frequent and at times severe or even fatal.

Most serious is symptomatic liver damage ranging from jaundice to hepatitis, which can be fulminant, severe liver necrosis, and liver cirrhosis. Also very important is a relatively high incidence of myelosuppression with leukopenia, and/or hypoplastic anemia, and/or thrombocytopenia. Infections, sometimes as severe as development of active tuberculosis, pneumonia, PCP, and severe viral or mycotical infections, possibly leading to sepsis, death or permanent damage have been seen. Anemia or bleeding episodes may also lead to serious complications.

\section{Interactions :}

Alcohol, other DMARDs including chloroquine/hydroxychloroquine, live virus vaccines, tegafur, some tuberculostatics (rifampin and/or isoniazid), tolbutamide and warfarin should not be given concomitantly.

\section{CONCLUSION:}

Most immune syndromes are caused by a deregulation in an auto regulating system. Conventional suppressive approach will often induce a switch of the balance, which means that the one rigidity is replaced by a new one, induced by the immunosuppressive medication itself. By using micro doses stimuli, coming from antihomotoxic medications, immunomodulation is possible in such a way that the rigidity changes into a balancing autoregulation again.

Immunomodulator can modulate immune systems in different ways with the use of exogenous or endogenous bioactive agents which utilize cell-to-cell signaling in order to reach homeostasis within an organism's innate cellular defense. Although immunomodulators are commonly thought to be suppressive agents, such biological activists are well known to offer immunostimulation. An extremely complex cellular symphony of signaling regulates an organism's immunostatus through immunomodulators.

\section{ACNOWLEDGMENT:}

Authors thank to Department of Pharmacy, Pranveer Singh Institute of Technology, Kanpur, India for their support and provide facilities. 


\section{REFERENCES :}

1. Immunomodulators Therapeutic Needs, Pipelines \& Prospects, Drug Discovery, by Biophoenix, 2007. P. 185-202.

2. Thappa DM, et al: Immunomodulators in the treatment of psoriasis, Indian J. Dermatol. Venereol. Leprol , 2004,70 (1), 3-5.

3. Fernando S. Velayos and William J. Sandborn, Current Gastroenterology Reports, 2007 (9), 521-527.

4. Richard E Gonsette, Advances in Treating Multiple Sclerosis, European neurological disease, 2007, 18-20.

5. Kappos L, Bates D, Hartung H P, et al., Natalizumab treatment for multiple sclerosis: recommendations for patient selection and monitoring, Lancet Neurol, 2007, 6, 431-441.

6. Zafar K. Khan and Pooja Jain, Antifungal Agents and Immunomodulators in Systemic Mycoses, The Indian Journal of Chest Diseases \& Allied Sciences, 2000, 42, 345-355.

7. R.C. Turowski, P.L. Triozzi, Application of chemical immunomodulators to the treatment of cancer and AIDS, Cancer Invest 1994, $12,620-43$.

8. Adams GP, Weiner LM., Monoclonal antibody therapy of cancer, Nat Biotechnol. 2005, 23(9), 1147-1157.

9. Sharad Mutalik, Treatment of keloids and hy pertrophic scars, Indian J Dermatol Venereol Leprol 2005, 71 (1), 3-8.

10. Siegel C., Hur C, Korzenik J, et al. Risks and benefits of infliximab for the treatment of Crohn's disease. Gastroenterology 2006; 130(4), A-71.

11. Wiwanitkit, V., Imiquimod: a new immunomodulatory drug. Chula. Med. J. 2004; 48(5), 325 - 338.

12. Steven K. Teo, Properties of Thalidomide and its Analogues: Implications for Anticancer Therapy, The AAPS Journal 2005, 7 (1), E14-E19.

13. Stefano Alcaro, Adriana Arena, et al., 9-Fluorenon-4-carboxamides: synthesis, conformational analysis, anti-HSV-2, and immunomodulatory evaluation, ARKIVOC 2004 (v), 334-348.

14. X de la Tribonnière, G Bahr, Murabutide: A synthetic immunomodulator under development as associated immunotherapy for HIV and HCV infections, Patentdocs, 16/12/02.

15. Fukushima R, Kanamori S, Hirashiba M, "Teratogenicity study of the dihydroorotate-dehy drogenase inhibitor and protein ty rosine kinase inhibitor Leflunomide in mice". Reprod. Toxicol. 200724 (3), 310-316. 